\title{
COVID 19: Relação do padrão epidemiológico da COVID-19 entre China e Itália
} COVID 19: Relationship of the epidemiological pattern of COVID-19 between China and Italy

\section{COVID 19: Relación entre el patrón epidemiológico de COVID-19 entre China e Italia}

Recebido: 23/05/2020 | Revisado: 26/05/2020 | Aceito: 27/05/2020 | Publicado: 11/06/2020

\section{Camilla Mandarine Ferreira}

ORCID: https://orcid.org/0000-0001-8817-9583

Faculdade de Medicina de Olinda, Brasil

E-mail: camillamandarine@yahoo.com.br

Denyse Diomedes Constantino de Almeida

ORCID: https://orcid.org/0000-0001-5589-9330

Faculdade de Medicina de Olinda, Brasil

E-mail: denysediomedes@ hotmail.com

\section{Manuela Lavareda Ayres Drummond de Mattos}

ORCID: https://orcid.org/0000-0003-4465-069X

Faculdade de Medicina de Olinda, Brasil

E-mail: ladymanuca@ hotmail.com

Thárcia Kiara de Beserra de Oliveira

ORCID: https://orcid.org/0000-0001-6352-7254

Faculdade de Medicina de Olinda, Brasil

E-mail: tharcia_kiara@hotmail.com

\section{Resumo}

O estudo teve como objetivo analisar o padrão epidemiológico da COVID-19 entre Itália e China. A pergunta que direcionou a revisão foi: Quais foram os fatores epidemiológicos que levaram à expressão da COVID-19 entre China e Itália? A busca pelos artigos fora realizada em abril de 2020 nas seguintes bases de dados: Scielo, Pubmed, BVS, ScienceDirect e Medline, utilizando os seguintes unitermos: Coronavirus infections, epidemiology e risk factors. Estudos demonstram notáveis diferenças da taxa de mortalidade entre populações asiáticas e europeias. Foram elencados três fatores de risco para o agravamento por COVID- 
19: sexo masculino, idade avançada (maior que 60 anos) e presença de comorbidades, principalmente as crônicas. Tais fatores mostraram-se predominantes principalmente na Itália, em comparação com a China. Hipertensão, diabetes, doenças cardiovasculares e doenças cerebrovasculares são consideradas como significantes fatores de risco para COVID-19 e seu agravamento. Estudos demonstram que para ambos os países, ser idoso e homem aumenta o risco de contrair a doença em sua forma mais grave. Faz-se necessário novos estudos mais aprofundados quanto aos efeitos maléficos das doenças crônicas como fatores de risco para a infecção pelo COVID-19.

Palavras-chave: Infecções por coronavírus; Epidemiologia; Fatores de risco.

\section{Abstract}

The study aimed to analyze the epidemiological pattern of COVID-19 between Italy and China. The question that guided the review was: What were the epidemiological factors that led to the expression of COVID-19 between China and Italy? The search for the articles was carried out in April 2020 in the following databases: Scielo, Pubmed, VHL, ScienceDirect and Medline, using the following keywords: Coronavirus infections, epidemiology and risk factors. Studies show notable differences in the mortality rate between Asian and European populations. Three risk factors for worsening due to COVID-19 were listed: male gender, advanced age (older than 60 years) and the presence of comorbidities, especially chronic ones. Such factors were predominant mainly in Italy, compared to China. Hypertension, diabetes, cardiovascular diseases and cerebrovascular diseases are considered to be significant risk factors for COVID-19 and its worsening. Studies show that for both countries, being elderly and male increases the risk of contracting the disease in its most severe form. Further, more in-depth studies are needed regarding the harmful effects of chronic diseases as risk factors for COVID-19 infection.

Keywords: Coronavirus infections; Epidemiology; Risk factors.

\section{Resumen}

El estudio tuvo como objetivo analizar el patrón epidemiológico de COVID-19 entre Italia y China. La pregunta que guió la revisión fue: ¿Cuáles fueron los factores epidemiológicos que llevaron a la expresión de COVID-19 entre China e Italia? La búsqueda de los artículos se realizó en abril de 2020 en las siguientes bases de datos: Scielo, Pubmed, VHL, ScienceDirect y Medline, utilizando las siguientes palabras clave: Infecciones por coronavirus, epidemiología y factores de riesgo. Los estudios muestran diferencias notables en la tasa de 
mortalidad entre las poblaciones asiáticas y europeas. Se enumeraron tres factores de riesgo de empeoramiento debido a COVID-19: género masculino, edad avanzada (mayores de 60 años) y la presencia de comorbilidades, especialmente crónicas. Tales factores fueron predominantes principalmente en Italia, en comparación con China. La hipertensión, la diabetes, las enfermedades cardiovasculares y las enfermedades cerebrovasculares se consideran factores de riesgo significativos para COVID-19 y su empeoramiento. Los estudios muestran que para ambos países, ser anciano y hombre aumenta el riesgo de contraer la enfermedad en su forma más grave. Además, se necesitan más estudios en profundidad sobre los efectos nocivos de las enfermedades crónicas como factores de riesgo para la infección por COVID-19.

Palabras-clave: Infecciones por coronavírus; Epidemiología; Factores de riesgo.

\section{Introdução}

Em Dezembro de 2019, diversos casos de pneumonia foram notificados na província de Wuhan, na China. Após pesquisas lideradas pelo médico Li Wenliang, foi descoberto que, na verdade, os casos tratavam de uma Síndrome Respiratória Aguda Grave associada ao coronavírus, provocada pelo SARS-COV tipo 2. Estudos apontam que o surto local esteja relacionado ao consumo de morcegos nos mercados de peixe, visto que o animal é reservatório natural de coronaviroses (Yan-Rong Guo et al., 2020; Ye Yi et al., 2020).

Por ser de alta virulência, a transmissão entre humanos dá-se de maneira rápida, através de contato com gotículas de tosse ou espirro de uma pessoa contaminada. Além de flutuar no ar na forma de aerossóis, o vírus permanece por algumas horas em locais cujas gotas respiratórias tenham caído e, caso um indivíduo suscetível entre em contato com os objetos contaminados e leve as mãos às mucosas e vias aéreas, também poderá contrair a infecção, tornando-se necessária a higienização do local afetado (Minah Parket et al., 2020; Yan-Rong Guo et al., 2020).

Em Janeiro de 2020, a OMS (Organização Mundial de Saúde) declarou que o surto de coronavírus constitui uma Emergência de Saúde Pública de Importância Internacional, elevando-a à condição de pandemia em 11 de Março de 2020, ao atingir aproximadamente 62 países. Atualmente, o SARS-COV 2 alcançou todos os continentes, exceto a Antártica, com mais de 85.000 óbitos ao redor do mundo, abrangendo mais de 190 países (OMS, 2020).

Desses 190 países afetados, a Itália é o país não asiático que mais vem sofrendo com a pandemia (Porcheddu et al., 2020). No dia 03 de fevereiro, 56 cidadãos italianos que 
estiveram em Wuhan foram diagnosticados com a doença e, a partir daí chegou a aproximadamente 80.500 casos confirmados e 8200 óbitos no final de Março de 2020, sendo a maioria da região da Lombardia. No entanto a China, que foi o epicentro da disseminação do vírus, registrou uma média de 81800 casos e 3296 óbitos na província até o dia 27 de Março de 2020, além de um elevado número de curados. Logo, é perceptível uma diferença de 1308 casos entre Itália e China e cerca de 4919 óbitos (Porcheddu et al., 2020; Gianfranco Spiteri et al., 2020).

Em comparação dos padrões epidemiológicos de casos entre China e Itália, as comorbidades parecem ser contribuintes importantes em morte e gravidade do COVID-19. Apesar de, nos dois países a faixa etária dos idosos de 60 anos ou mais na Itália e mais de $50 \%$ idades superior a 50 anos na China, mostra semelhança do acometimento na maioria dos idosos com comorbidades conhecidas (Remuzzo et al., 2020).

É perceptível que há uma semelhança no padrão epidemiológico entre China e Itália, no entanto na Itália houve uma menor infectividade viral, porém maior letalidade, pois a Itália retratou mais de 7.000 mortes em um mês após o primeiro óbito do país, ultrapassando os números da China. Com isso, este trabalho teve como objetivo analisar o padrão epidemiológico da COVID-19 entre Itália e China.

\section{Metodologia}

Trata-se de um estudo de revisão de literatura que revisa rigorosamente e combina estudos com diversas metodologias, comparando a ocorrência da doença entre grupos de pessoas seguindo as orientações encontradas em Evidências Científicas. A pergunta que direcionou a revisão foi: Quais foram os fatores epidemiológicos que levaram à expressão da Covid-19 entre China e Itália?

\section{Estratégia de busca}

A busca pelos artigos fora realizada em abril de 2020 nas seguintes bases de dados: Scielo, Pubmed, BVS,ScienceDirect e Medline, utilizando os seguintes unitermos: Coronavirus infections, epidemiology e riskfactors. Houve restrição em artigos cujo idioma não fosse o inglês.

$\mathrm{Na}$ fase inicial foi realizada uma leitura dos títulos e/ou abstracts aplicando os critérios de seleção. Após esta fase, os revisores independentes executaram uma leitura previa de todos os 
artigos selecionados. Os resultados das pesquisas encontram-se sumarizados no fluxograma da Figura 1.

Figura1: O processo de seleção dos artigos para busca.

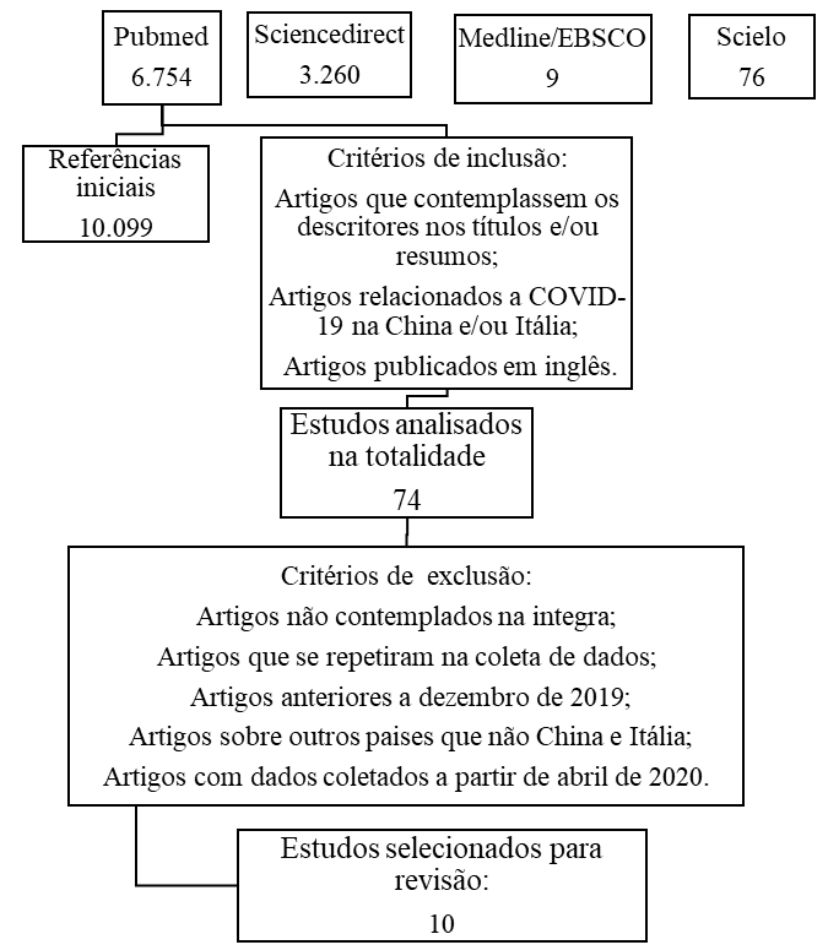

Fonte: Autores 2020.

O estudo contemplou os seguintes critérios de inclusão: a) Artigos relacionados ao COVID-19 na China e/ou Itália; b) Artigos que contemplassem os descritores nos títulos e/ou resumos; c) Artigos publicados em inglês; d) Artigos que relacionassem a COVID-19 ao sistema respiratório. Foram excluídos: a) Artigos não contemplados na íntegra; b) Artigos que se repetiram na coleta de dados; c) Artigos anteriores a dezembro de 2019; d) Artigos sobre outros países que não China e Itália; e) Artigos com dados coletados a partir de abril de 2020.

\section{Resultados e Discussão}

As estratégias de busca permitiram a identificação de 10.099 artigos. Destes, apos serem inclusos os criterios de inclusão e exclusão, foram selecionados 10 estudos (Figura 1). 
Quadro 1: Publicações incluídas na revisão de literatura, segundo o objetivo do estudo.

\begin{tabular}{|c|c|c|c|c|}
\hline & TÍTULO & AUTOR & OBJETIVOS & CONCLUSÃO \\
\hline $1^{\circ}$ & $\begin{array}{l}\text { Clinical and demographic } \\
\text { characteristics of patients } \\
\text { dying from } \\
\text { covD19 in Italy versus } \\
\text { China }\end{array}$ & Lippi et al & $\begin{array}{l}\text { Comparar o impacto da } \\
\text { incidência de mortalidade } \\
\text { por COVID-19 entre } \\
\text { China e Itália e as } \\
\text { variáveis de taxa. }\end{array}$ & $\begin{array}{l}\text { Sexo masculino, idade avançada ( }>60 \text { anos) e a } \\
\text { presença de comorbidades foram os fatores discutidos } \\
\text { que aumentam o risco de mortalidade por COVID-19. }\end{array}$ \\
\hline $2^{\circ}$ & $\begin{array}{l}\text { Epidemiological and clinical } \\
\text { features of } 125 \text { Hospitalized } \\
\text { Patients with COVID-19 in } \\
\text { Fuyang, Anhui, China }\end{array}$ & Wang et al & $\begin{array}{l}\text { Elencar as diferenças } \\
\text { epidemiológicas entre } \\
\text { pacientes na província de } \\
\text { Wuhan e fora dela. }\end{array}$ & $\begin{array}{l}\text { A febre foi retratada como o sintoma mais comum. } \\
\text { Idade elevada, doenças crônicas e histórico de } \\
\text { tabagismo são fatores que levam ao agravamento da } \\
\text { condição. }\end{array}$ \\
\hline $3^{\circ}$ & $\begin{array}{l}\text { Clinical progression of patients } \\
\text { with COVID-19 in Shanghai, } \\
\text { China }\end{array}$ & Chen et al & $\begin{array}{l}\text { Destrinchar o progresso } \\
\text { clínico do coronavírus. }\end{array}$ & $\begin{array}{l}\text { Sexo masculino, idade elevada e alterações em } \\
\text { exames laboratoriais estão relacionados à maior } \\
\text { chance de desenvolver angústia respiratória. }\end{array}$ \\
\hline $4^{\circ}$ & $\begin{array}{l}\text { Whole genome and } \\
\text { phylogenetic analysis of two } \\
\text { SARS-CoV-2 strains isolated in } \\
\text { Italy in January and February } \\
\text { 2020: additional clues on } \\
\text { multiple introductions and } \\
\text { further circulation in Europe }\end{array}$ & $\begin{array}{l}\text { Stefanelli et } \\
\text { al }\end{array}$ & $\begin{array}{l}\text { Compreender a } \\
\text { epidemiologia molecular } \\
\text { da pandemia na Itália. }\end{array}$ & $\begin{array}{l}\text { A mutação identificada na Lombardia, comparada ao } \\
\text { código genético do vírus de Wuhan, deve ser mais } \\
\text { bem investigada a fim de melhor entender o que } \\
\text { afeta as características virais para que haja um } \\
\text { potencial de mutação elevado. }\end{array}$ \\
\hline $5^{\circ}$ & $\begin{array}{l}\text { Differential diagnosis of illness } \\
\text { in patients under investigation } \\
\text { for the novel coronavirus } \\
\text { (SARS-CoV-2) }\end{array}$ & Bordi et al & $\begin{array}{l}\text { Comparar diagnósticos } \\
\text { diferenciais do } \\
\text { coronavírus com doenças } \\
\text { semelhantes. }\end{array}$ & $\begin{array}{l}\text { As diferenças percebidas entre os chineses e italianos } \\
\text { relacionam-se à imunidade do hospedeiro, assim } \\
\text { como a exposições prévias ao vírus. Diagnóstico } \\
\text { diferencial com infecções comuns deve ser realizado, } \\
\text { pois há uma similaridade de sintomas. }\end{array}$ \\
\hline $6^{\circ}$ & $\begin{array}{l}\text { Similarity in Case Fatality } \\
\text { Rates (CFR) of COVID- } \\
\text { 19/SARS-COV-2 in Italy and } \\
\text { China }\end{array}$ & $\begin{array}{l}\text { Porcheddu } \\
\text { et al }\end{array}$ & $\begin{array}{l}\text { Descrever a similaridade } \\
\text { de casos graves e } \\
\text { mortalidade entre China } \\
\text { e Itália. }\end{array}$ & $\begin{array}{l}\text { A severidade da doença e mortalidade encontram-se } \\
\text { associadas, tanto na China quanto na Itália, à idade } \\
\text { mais elevada e comorbidades. Mais de } 50 \% \text { das } \\
\text { fatalidades da China ocorreram em pessoas }>50 \\
\text { anos. Na China, poucas pessoas }<19 \text { anos morrem (1 } \\
\text { em 965). }\end{array}$ \\
\hline $7^{\circ}$ & $\begin{array}{l}\text { As COVID-19 cases, deaths } \\
\text { and fatality rates surge in Italy, } \\
\text { underlying causes require } \\
\text { investigation }\end{array}$ & $\begin{array}{l}\text { Rubino et } \\
\text { al }\end{array}$ & $\begin{array}{l}\text { Investigar a disparidade } \\
\text { da taxa de mortalidade } \\
\text { entre China e Itália } \\
\text { através de dados } \\
\text { epidemiológicos e } \\
\text { fatores de risco. }\end{array}$ & $\begin{array}{l}\text { Os avanços no manejo de doenças cardiovasculares } \\
\text { nos últimos } 20 \text { anos levaram a uma população } \\
\text { bastante fértil para o SARS Cov-2 na Itália, visto que } \\
\text { muitos não se previnem devido à elevada } \\
\text { disponibilidade de medicamentos. } 90 \% \text { dos mortos } \\
\text { possuíam } 1 \text { doença de base e alteração } \\
\text { cardiovascular foi encontrada em mais de } 70 \% \text { dos } \\
\text { pacientes. }\end{array}$ \\
\hline $8^{\circ}$ & $\begin{array}{l}\text { Coronavirus Disease (COVID- } \\
\text { 19) in Italy: Analysis of Risk } \\
\text { Factors and Proposed Remedial } \\
\text { Measures }\end{array}$ & $\begin{array}{l}\text { Lorenzo et } \\
\text { al }\end{array}$ & $\begin{array}{l}\text { Elencar fatores que } \\
\text { identifiquem a elevada } \\
\text { letalidade na Itália }\end{array}$ & $\begin{array}{l}\text { A maioria das mortes italianas consistem em homens } \\
\text { na faixa dos } 79 \text { anos, enquanto na Ásia em mulheres } \\
\text { jovens. Outra explicação é a presença de } \\
\text { comorbidades na população idosa e a contaminação } \\
\text { através de assintomáticos. }\end{array}$ \\
\hline $9^{\circ}$ & $\begin{array}{l}\text { Risk Factors Associated With } \\
\text { Acute Respiratory Distress } \\
\text { Syndrome and Death in } \\
\text { Patients With Coronavirus } \\
\text { Disease } 2019 \text { Pneumonia in } \\
\text { Wuhan, China }\end{array}$ & Wu et al & $\begin{array}{l}\text { Descrever as } \\
\text { características clínicas e } \\
\text { desfecho dos pacientes } \\
\text { diagnosticados com } \\
\text { COVID-19 que } \\
\text { desenvolveram síndrome } \\
\text { respiratória ou } \\
\text { morreram. }\end{array}$ & $\begin{array}{l}\text { Idade avançada foi associada com maior risco de } \\
\text { desenvolver angústia respiratória e de morte devido à } \\
\text { redução da resposta imune por parte do paciente. } \\
\text { Neutrofilia relaciona-se à síndrome respiratória, } \\
\text { assim como disfunção de órgãos e de coagulação e } \\
\text { pioram o prognóstico. }\end{array}$ \\
\hline 10 & $\begin{array}{l}\text { Severe acute respiratory } \\
\text { syndrome (SARS) and } \\
\text { coronavirus disease-2019 } \\
\text { (COVID-19): From causes to } \\
\text { preventions in Hong Kong }\end{array}$ & Law et al & $\begin{array}{l}\text { Resumir o conhecimento } \\
\text { obtido sobre o } \\
\text { coronavirus e comparar } \\
\text { com experiências prévias } \\
\text { da epidemia de SARS. }\end{array}$ & $\begin{array}{l}\text { Higiene pessoal e medidas de proteção, como a } \\
\text { adoção de máscaras e luvas são as formas mais } \\
\text { importantes e simples de reduzir o contágio pela } \\
\text { doença, assim como evitar aglomerações e aderir ao } \\
\text { isolamento social. }\end{array}$ \\
\hline
\end{tabular}

Fonte: Autores 2020.

Estudos demonstram notáveis diferenças da taxa de mortalidade entre populações asiáticas e europeias. Foram elencados três fatores de risco para o agravamento por COVID- 
19: sexo masculino, idade avançada (maior que 60 anos) e presença de comorbidades, principalmente as crônicas. Tais fatores mostraram-se predominantes principalmente na Itália, em comparação com a China (Lippi et al., 2020). Uma possível explicação para a diferença do padrão de agressividade do vírus de uma região para a outra é a maior testagem e relatórios epidemiológicos providenciados pelos Governos locais. Logo, é recomendada uma maior proteção individual a fim de evitar a disseminação da doença, como o distanciamento social.

A febre é o sintoma mais comum entre os chineses, acompanhada de tosse, fadiga, dor de garganta e mialgia. Indivíduos de todas as idades são suscetíveis, mas o agravamento dá-se principalmente em pessoas de meia idade, sendo mais frequente em homens devido aos hábitos de vida, tal qual o fumo, que aumenta a expressão da enzima conversora de angiotensina 2 no tecido pulmonar, a qual é a receptora do vírus (Wang et al., 2020)

A alteração em exame radiológico mais comum é o padrão de vidro-fosco à tomografia computadorizada. Diagnóstico precoce e tratamento têm um efeito positivo no prognóstico, enquanto idade avançada, doença crônica de base e fumo levam a um pior prognóstico, segundo dados colhidos por Wang et al. (2020).

(Chen et al., 2020) descreveu primeiramente 41 casos de COVID-19 em pacientes que se alimentaram no mercado de frutos do mar de Wuhan, por meio de trabalho produzido por (Wang et al., 2020). (Chen et al., 2020) e (Wang et al., 2020) relataram 99 e 138 casos de COVID em Wuhan, respectivamente, sugerindo que pacientes idosos com comorbidades tendem a sofrerem com depressão respiratória.

O estudo de (Stefanelli et al., 2020) faz uma comparação entre duas cepas de SARSCov-2, na qual uma foi de um chinês que estava visitando Roma e a outra foi de um italiano. Os dois pacientes foram hospitalizados com pneumonia, ambos apresentaram comprometimento pulmonar bilateral com opacidade em vidro fosco. O paciente chinês passou 10 dias após o início dos sintomas para ser internado, enquanto o italiano foi internado no mesmo dia que apresentou em que se tornou sintomático. A sequência das duas cepas mostrou que o genoma do turista chinês diferiu em duas posições "nt" da do paciente em Wuhan, sendo agrupada com cepa europeia e cepa da Austrália, enquanto a sequência do paciente italiano apresentou quatro variações "nt", agrupando-se com cepa da Alemanha e México. Foi observado, portanto que há mutações virais, fazendo com que o vírus da china se diferencie do vírus na Itália.

Sobre o diagnóstico diferencial em pacientes sob investigação para COVID-19, o estudo de (Bordi et al., 2020) afirma que os sintomas são semelhantes a outras doenças respiratórias e o diagnóstico diferencial deve incluir infecções comuns, como influenza e 
outras. Foi feito um estudo com casos suspeitos e com histórico recente de viagem à Ásia ou que teve contato com algum caso confirmado, no qual a idade média foi de 35 anos. Desses 126 pacientes, apenas três foram positivos para SARS-CoV-2, podendo então observar a necessidade de fazer o diagnóstico diferencial com as outras infecções de trato respiratório.

(Porcheddu et al., 2020) realizaram uma investigação da similaridade dos casos fatais da China e Itália. Os primeiros casos relacionados a viagem, onde 56 cidades italianos que estavam em Wuhan, na China, dentre eles havia um estudante de 17 anos que estava com febre e não foi permitido no voo e um pesquisador de 29 anos, o qual desenvolveu sintomas do COVID-19. O navio Diamond Princess, que desembarcou em Hong Kong, foi colocado de quarentena pois um passageiro testou positivo para COVID-19 e dentre 3700 pessoas a bordo do navio, 35 eram italianos e um deles positivou para o vírus. Os primeiros casos em Roma eram turistas chineses de Wuhan, dois idosos que foram confirmados como positivos. Nesses estudos, foi sugerido que o paciente zero na região italiana tenha sido um homem de 38 anos que apresentou uma infecção respiratória grave após contato com um amigo que esteve na China.

(Rubino et al., 2020) investigaram como os casos de COVID-19 levaram às elevadas taxas de mortalidade na Itália. Em termos de taxas de mortalidade por casos (CFR), a Itália estaria com $10,6 \%$ e a China com $4 \%$, visando um grande diferencial do número absoluto de fatalidades e as grandes diferenças na CFR. Foi necessária a busca de causas subjacentes.

(Law et al., 2020) revisaram as causas, transmissões, sintomas e medidas preventivas do SARS e COVID-19 em Hong Kong, deixando diagnóstico e tratamento para estudos futuros. Nesse estudo, relacionaram a causa do COVID-19 à infecção por coronavírus ocorrida no mercado atacadista de frutos do mar e animais em Wuhan, na China. A transmissão da SARS-Cov 2 em humano- humano é através de gotículas ou contato direto. $\mathrm{O}$ período de incubação para COVID-19 se expande para 5 dias e a quarentena é de 14 dias a partir da última data de exposição. Os transtornos sintomáticos revelaram ser febre, tosse e fadiga, além dos distúrbios respiratórios como rinorreia, espirros, dor de garganta e pneumonia. Relataram não existir um regime de tratamento específico e nenhuma vacina para prevenção no momento, mas a higiene pessoal e proteção com máscaras e lavar as mãos, bem como reduzir contato físico e multidões, se mostraram eficazes para impedir a disseminação do COVID- 19.

A taxa de letalidade do COVID-19 é maior na Itália (9\%) do que em Wuhan (5,8\%), de acordo com o estudo de (Lorenzo et al., 2020). Acredita-se que essa diferença ocorre devido à alta idade média italiana comparada a alguns locais na China. Esse trabalho afirma 
qua a maioria dos casos na República da Coréia são de mulheres jovens (62\%), na faixa etária de 20-30 anos (30\%), enquanto na Itália o predomínio é de 79 anos e mais de $70 \%$ eram homens. O trabalho supôs que as taxas informadas sobre a letalidade na Itália sejam inferiores, pois os números de infecções podem estar sendo subnotificados.

(Wu et al., 2019), confirma que idade avançada, neutrofilia e disfunção de órgãos e coagulação são fatores de risco para o desenvolvimento da coronavirose e sua possível progressão. Foram coletados dados de 201 pacientes até 13 de fevereiro de 2020, podendo perceber que células TCD4 e CD8 podem proteger os pacientes de desenvolver quadros mais avançados, mas devido ao tamanho limitado da amostra não foi possível observar as vantagens dessas células quanto à diminuição das mortes.

Logo, tendo como base os estudos discutidos, foi notável uma semelhança entre a epidemiologia da Itália e da China com relação ao agravamento sintomatológico no sexo masculino e em pacientes com uma ou mais comorbidades crônicas. Tal fato deve-se à maior depressão do sistema imune apresentada por esses indivíduos, além da elevada infectividade do vírus e de sua taxa de transmissibilidade.

\section{Considerações Finais}

O COVID-19 é, atualmente, considerado um dos principais problemas de saúde pública mundial, sendo caracterizado como uma nova pandemia, pois afetou mais de 80 países no mundo. Através dessa revisão integrativa de literatura, foi possível identificar hipertensão, diabetes, doenças cardiovasculares e doenças cerebrovasculares como significantes fatores de risco para COVID-19 e seu agravamento, bem como um aspecto epidemiológico característico na China e Itália. Alguns estudos têm caracterização de padrão, como pacientes jovens do sexo feminino na China e paciente idosos do sexo masculino na Itália, porém a maioria demonstra que, para ambos países, ser idoso e homem aumenta o risco de contrair a doença em sua forma mais grave.

Contudo, ainda há uma escassez de artigos publicados relacionados ao assunto, sendo válidas futuras pesquisas a fim de comprovar essa interação epidemiológica não só entre China e Itália, mas também todos os países contaminados por essa pandemia, principalmente com relação às comorbidades presentes em pacientes com coronavírus. Logo, faz-se necessários novos estudos mais aprofundados quanto aos efeitos maléficos das doenças crônicas como fatores de risco para a infecção pelo COVID-19 e aos aspectos 
epidemiológicos que contribuíram para a elevada taxa de mortalidade pela doença, principalmente na Itália.

\section{Referências}

Bordi L., Nicastri E., Scorzolini L., Caro A.D., Capobianchi M.R., Castilletti C. \& Lalle E. (2020). Differential Diagnosis of Illness in Patients Under Investigation for the Novel Coronavirus (SARS-CoV-2). (2020). Italy, February. Eurosurveillance. Feb 27; 25,(8). 2000170

Chen J., Qi, T., Liu L., Ling Y., Qian Z., Li, T. \& Lu H. (2020).Clinical progression of patients with COVID-19 in Shanghai, China.Journal of Infection. 80,(20). 1-6.

Guo Y.R., Cao Q.D., Hong Z.S., Tan Y.Y., Chen S.D., Jin H.J., Tan K.S. \& Wang D.Y. (2020). The origin, transmission and clinical therapies on coronavirus disease 2019 (COVID19) outbreak - an update on the status.Military Medical Research. 7,(11). 1-10.

Law S., Leung A.W. \& Xu C. (2020). Severe acute respiratory syndrome (SARS) and coronavirus disease-2019 (COVID-19): From causes to preventions in Hong Kong. Int J Infect Dis.4,(94). 156-163.

Lippi G., Mattiuzzi C., Sanchis-Gomar F. \& Henry,B.M. (2020). Clinical and demographic characteristics of patients dying from COVID-19 in Italy versus China. 11,(4). 1-9.

Lorenzo G.D. \& Trolio R.D. (2020). Coronavirus Disease (COVID-19) in Italy: Analysis of Risk Factors and Proposed Remedial Measures. Front in Medicine. 7,(140).

Organização Pan-Americana de Saúde. (2020). Folha informativa - COVID-19 (doença causada pelo novo coronavírus). Acesso em 15 de abril, Disponível em: https://www.paho.org/bra/index.php?option=com_content\&view=article\&id=6101: covid19\&I temid $=875$ 
Park M., Cook A.R., Yinxiaohe J.T. \& Dickens B.L. (2020). A Systematic Review of COVID-19 Epidemiology Based on Current Evidence. Journal of Clinical Medicine. 9, (4), 967-73.

Porcheddu R. Serra C., Kelvin D., Kelvin N. \& Rubino S. (2020). Similarity in Case Fatality Rates (CFR) of COVID-19/SARS-COV-2 in Italy and China.The Journal of Infection in Developing Countries.14, (2).1-4.

Remuzzi A. \& Remuzzi G. (2020). Covid-19 and Italy: what next? The lancet. 11.(395), 1225-1228.

Rubino, S., Kelvin, N., Bermejo-Martin, J.F. \& Kelvin, D.J. (2019). As COVID-19 cases, deaths and fatality rates surge in Italy, underlying causes require investigation. Infect DevCtries. 14,(3). 265-267.

Spiteri G., Fielding J., Diercke M., Campese C., Enouf, V., Gaymard A., Bella, A., Sognamiglio, P., Moros M.J.S. \& Riutort A.N. (2020).First cases of coronavirus disease 2019 (COVID-19) in the WHO European Region, 24 January to 21 February 2020.Euro Surveill. 25,(9). 200- 178.

Stefanelli, P., Faggioni, G., Presti, A.L., Fiore, S., Marchi, A., Benedetti, E. \& Lista, F. (2020). Whole genome and phylogenetic analysis of two SARS-CoV-2 strains isolated in Italy in January and February 2020: additional clues on multiple introductions and further circulation in Europe.(2020).Euro Surveill. 25,(13). 200-305

Wang R., Pan M., Zhang X., Han M., Han M., Fan X. \& Shen L. (2020). Epidemiological and clinical features of 125 Hospitalized Patients with COVID-19 in Fuyang, Anhui, China. Int J Infect Dis. Apr 11,(95). 421-428.

World Health Organization.(2020). SARS (Severe Acute Respiratory Syndrome).Acessoem 15 de abril, Disponível em: https://www.who.int/ith/diseases/sars/en/

Wu C., Chen X., Cai., Y., Xia J., Zhou X., Xu S. \& Song Y. (2020). Risk Factors Associated With Acute Respiratory Distress Syndrome and Death in Patientes With Coronavirus Disease 
2019 Pneumonia in Wuhan China. JAMA Intern Med. Retrieved from https://jamanetwork.com/journals/jamainternalmedicine/fullarticle/2763184

Yi Y., Lagniton P.N.P., Ye S., Li E. \& Xu R.H. (2020). COVID-19: what has been learned and to be learned about the novel coronavirus disease. International Journal of Biological Sciences.16,(10). 1753-1766.

\section{Porcentagem de contribuição de cada autor no manuscrito}

Camilla Mandarine Ferreira-30\%

Denyse D Constantino de Almeida - 25\%

Manuela Lavareda D de Mattos- 25\%

Thárcia Kiara de Beserra de Oliveira-20\% 